\title{
Importance of a Multidisciplinary Approach to Breast Cancer Treatment in Pregnancy: Case Report of New Diagnosis of Pregnancy-Associated Breast Cancer
}

\author{
Grant $\mathrm{J}^{1}$, DiCarlo $\mathrm{C}^{2}$ and Woessner-Hoyson $\mathrm{J}^{1^{*}}$ \\ ${ }^{1}$ Department of Obstetrics and Gynecology, Western Pennsylvania Hospital, Pittsburgh, Pennsylvania, USA \\ ${ }^{2}$ Department of Pathology, Allegheny General Hospital, Pittsburgh, Pennsylvania, USA \\ "Corresponding author: Woessner-Hoyson J, Department of Obstetrics and Gynecology, Western Pennsylvania Hospital, Pittsburgh, Pennsylvania, USA, Tel: \\ 724-742-3839; E-mail: Jessica.hoyson@ahn.org
}

Received date: November 23, 2016; Accepted date: December 12, 2016; Published date: December 16, 2016

Copyright: $\odot 2016$ Grant J, et al. This is an open-access article distributed under the terms of the Creative Commons Attribution License, which permits unrestricted use, distribution, and reproduction in any medium, provided the original author and source are credited.

\begin{abstract}
Pregnancy-Associated Breast Cancer (PABC) is a rare diagnosis and includes new diagnoses of cancer both during pregnancy as well as within the first year post-delivery. Due to its rarity, there is of yet no gold standard treatment nor is there a standardized regimen of treatment during pregnancy according to the American College of Obstetrics and Gynecology (ACOG). We report a case involving a 35-year-old gravida 2 para 1-0-0-1 who was diagnosed with clinical stage II (T2 N1) breast cancer in the early third trimester of pregnancy after physical examination revealed a palpable mass. Ultrasound-guided biopsy revealed poorly differentiated infiltrating ductal carcinoma, nuclear grade 3, with micropapillary features, estrogen receptor (ER 90\%), progesterone receptor (PR $25 \%$ positive, HER2 positive $3+$ with Ki67 index $75 \%$. After extensive counseling and discussion between Obstetrics, Maternal Fetal Medicine, Breast Surgery, Neonatal ICU, and Oncology, a decision was made to initiate neoadjuvant chemotherapy (NAC) with adriamycin and cyclophosphamide. Our patient completed 4 total NAC treatments prior to delivery followed by a regimen of weekly taxol plus herceptin and perjeta postpartum. This patient strongly desired to carry the pregnancy to term and began treatment prior to delivery, making this case unique in comparison to other publications in which treatment was delayed until after delivery, or the pregnancy was terminated prior to beginning treatment. Our case highlights the importance of a multi-disciplinary approach to counseling patients in this unique situation to allow them the autonomy to choose the treatment best for them and their baby.
\end{abstract}

Keywords: Breast cancer; Benign eczematous lesion; Chemotherapy

\section{Introduction}

Breast cancer is the second most common malignancy diagnosed during pregnancy after cervical cancer, comprising approximately $0.2 \%-2.6 \%$ of all breast cancers $[1,2]$. The incidence of breast cancer in pregnancy is reported to be between 1 in 3000 and 1 in 10,000 pregnancies. As more women delay childbearing, the incidence of breast cancer in pregnancy is expected to increase, with women over the age of 35 being at greatest risk [3-5].

The diagnosis of breast cancer is a difficult one to discern due to physiologic changes of the breast that occur in pregnancy including hypertrophy, engorgement, nodularity, and discharge. This can result in pregnant women being diagnosed in advanced stages and having poorer prognoses upon diagnosis in comparison to non-pregnant women due to a delay in diagnosis. In a case study by Nargota, et al. a woman with Paget's disease was not diagnosed until one year after delivery given the difficulty in diagnosis as Paget's of lactation can be mistaken as a benign eczematous lesion [6].

Current recommendations for initial prenatal physical examinations include a complete bilateral breast examination which should be repeated at the 6 week postpartum visit as well as any time throughout the antepartum period in which the patient notices a change on selfexamination [4]. Breast masses encountered in pregnancy are mainly benign, with the differential diagnosis including abscess, lipoma, lactating adenoma, fibrocystic disease, hamartoma, and milk retention cyst. Despite its rarity, clinicians must have a high index of suspicion for malignancy. A breast mass that is persistent for greater than 2 weeks should be further evaluated with imaging $[1,7]$. Any palpable breast mass that shows atypical or suspicious features either clinically or on imaging, must undergo cytological and histological evaluation with a core biopsy $[1,6]$.

\section{Case Report}

A 35-year-old Caucasian woman (gravida 2 para 1-0-0-1) presented to the gynecology office at $303 / 7$ weeks gestation with the complaint of a newly palpable lump in her right breast which she noticed while showering. Initial physical examination performed at her initial prenatal visit (9 5/7 weeks gestation) revealed no abnormalities. On repeat examination, however, the right breast contained a $2 \times 2 \mathrm{~cm}$ freely mobile mass at the 9 o'clock position. There were no notable retractions or skin changes, nor any axillary lymphadenopathy appreciated. She had denied any trauma to the area or constitutional symptoms. Family history was pertinent for early breast cancer in her paternal grandmother. She underwent a breast sonogram which revealed a $2.5 \times 1.2 \times 2.9 \mathrm{~cm}$ mass at 9 oclock position with a 2 nd hypoechoic mass located at the 8 o'clock position measuring $1.3 \times 1.1 \times$ $1.2 \mathrm{~cm}$. A mammogram confirmed the masses and also showed microcalcifications in the entire right upper quadrant of the right breast (Figure 1). Ultrasound-guided biopsy revealed poorly 
differentiated infiltrating ductal carcinoma, nuclear grade 3, with micropapillary features, estrogen receptor (ER 90\%), progesterone receptor (PR 25\%) positive, HER2 positive 3+ with Ki67 index 75\% (Figure 2). We were able to arrange a meeting with Maternal Fetal Medicine (MFM) when patient was 32 3/7 weeks gestation for delivery planning. At that visit, fetal surveillance was performed. Fetal growth was $53^{\text {rd }}$ percentile (2114 grams) with biophysical profile (BPP) 8/8 and amniotic fluid index (AFI) of $19.79 \mathrm{~cm}$. Recommendations were dose dense neoadjuvant therapy with adriamycin and cyclophosphamide every 2-3 weeks for 4 treatments, followed by weekly taxol and herceptin plus perjeta every 3 weeks, with full year of herceptin. BioFlor port catheter was placed by invasive radiology at 32 $4 / 7$ weeks gestation, and she underwent two cycles consisting of adriamycin and cytoxan at $334 / 7$ and $354 / 7$ weeks gestation with supportive medications that included zofran, methylprednisolone, emend, and neulasta. After a two-week chemo washout, she presented for induction at 37 3/7 weeks gestation. Admission complete blood count (CBC): white blood count 12.9, hemoglobin 10.6, hematocrit 32.5 , with platelets 218 . Induction resulted in a normal spontaneous vaginal delivery of a viable female infant with APGARs (appearance, pulse, grimace, activity, respirations) of 8 at 1 minute and 9 at 5 minutes. She resumed chemotherapy with the third cycle administered one week following her delivery after she had had the appropriate time to heal. Her fourth and final treatment of Adriamycin and Cytoxan was given three weeks later followed by 15 total treatments of weekly taxol with herceptin plus perjeta every 3 weeks to complete her neoadjuvant chemotherapy. Due to the extent of tumor involvement and microcalcifications, mastectomy was planned. Patient then underwent bilateral mastectomies, right sentinel lymph node biopsy, and bilateral direct-to-implant reconstruction and alloderm placement upon completion of neoadjuvant systemic chemotherapy and after positron emission tomography - computed tomography (PET-CT) showed no evidence of distant metastases. Final pathology from her surgery showed a residual $1.4 \mathrm{~cm}$ right breast tumor of high nuclear grade 4 and ER/PR positive and negative right axillary lymph nodes. According to AJCC pathologic TND staging for invasive breast cancer, this is ypT1c, ypN0, pMX. Following surgery, the patient continued adjuvant chemotherapy with Herceptin every 3 weeks. Given the positive hormone status, this patient was then started on tamoxifen therapy which she continues at this time. Currently, this patient is being evaluated for the possible use of adjuvant radiation therapy prior to starting Lupron and arimidex therapy.

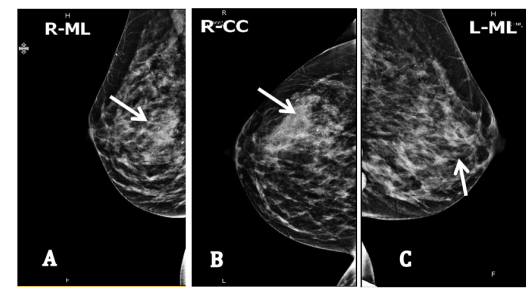

Figure 1: A and B Right breast proven carcinomas at 9:00 and 8:00 positions with associated extensive pleomorphic malignant calcifications in the outer upper and lower breast (arrow) extending from nipple to posterior breast. C The far right film shows an indeterminate group of calcifications in the lower posterior left breast (arrow).

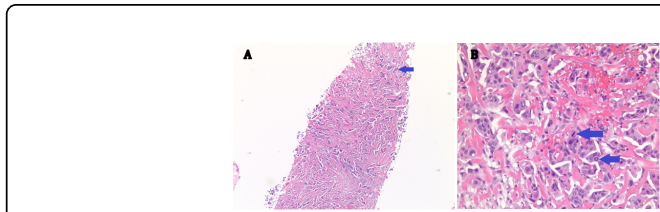

Figure 2: Poorly differentiated invasive ductal carcinoma of the breast with micropapillary features. A) (Left), Tumor cells are arranged in small clusters surrounded by empty, clear spaces in a fibrocollagenous background (arrow). Glandular formation is less than 10\% (H\&E, 40X). B) (Right), High-power magnification demonstrates a high nuclear grade with significant nuclear pleomorphism and frequent mitoses illustrated by the arrows (H\&E, 200X).

\section{Discussion}

Given the trend of delayed childbearing in women as time progresses, pregnancy-associated breast cancer, once a rare disease, is becoming more frequently observed. Because of this, it is imperative that a gold standard of care be adapted by all sub-divisions of the medical community, including Obstetrics and Gynecology given that in the cohort in question, initial diagnosis and work up will be performed by the obstetrician. As of yet there are no definitive regimens of care in the obstetrical guidelines for this growing cohort of patients, and recommendations at this time appear to be to treat each case uniquely taking into account all aspects including gestational age, aggression of disease, patient preference. Literature review has revealed that studies to date have focused heavily on two major cohorts: those women who are early in gestation and elect a termination of pregnancy while in the first or early second trimester prior to starting treatment, and those women who are diagnosed later in pregnancy and elect to delay treatment until after delivery of the fetus. This dichotomous categorization of women is starting to come into question.

In a study performed by Shim, et al. 87 women treated for various malignancies during pregnancy were monitored. These cancers included breast, gastrointestinal, hematologic, thyroid, central nervous system, cervical, ovarian, and lung amongst others. Out of 20 total women with breast cancer, $15 \%$ elected termination of pregnancy prior to treatment, $25 \%$ elected iatrogenic preterm delivery prior to treatment, $10 \%$ delayed treatment until after spontaneous delivery, and $50 \%(\mathrm{~N}=10)$ underwent treatment during pregnancy. While the description of said treatment was not identified, this study did validate that therapeutic abortion or termination of pregnancy was not necessary for all patients prior to initiating cancer treatment [8]. Not only is termination not necessary, but some studies have also demonstrated a lack of survival benefit to the patient with termination of pregnancy prior to initiation of cancer treatment $[1-4,7]$.

Additionally, several studies have highlighted the importance of avoiding premature delivery whenever possible. Preterm delivery can lead to acute complications, including neonatal or infant death, and has an important impact on neurophysiological outcome. These risks of complications increase with decreasing gestational age. The diagnosis of breast cancer in pregnancy represents a critical clinical situation however it is not an emergency. Lambertini, et al. concluded that there is usually enough time to use a multidisciplinary approach regardless of the gestational age at which diagnosis is made. However, despite the amount of data on chemotherapy use during pregnancy, the 
review noted that treatment recommendations of these women rely on limited evidence and that there were no specific guidelines for monitoring individuals with prenatal exposure to agents [1,9-11].

Another study by Amant, et al. showed a similar overall survival rate between women diagnosed with PABC and women with breast cancer diagnosed outside of pregnancy. This study involved a total of 311 eligible patients from around Europe with a median follow up period of 61 months. They looked at various types of chemotherapy, surgery, radiation treatment, and endocrine treatment in these cohorts with several subgroup analyses evaluated. Although overall survival (OS) and disease-free survival (DFS) were slightly higher in the nonpregnant patients when taken as a whole, there was no difference in OS or DFS when pregnant patients were compared with nulliparous nonpregnant patients. Data also suggested that survival rates did not statistically differ between those pregnant patients who opted for treatment prior to delivery and those patients who postponed treatment until the postpartum period [2].

As treatment for patients with breast cancer in pregnancy is noted to be similar to that of non-pregnant women, it is recommended that therapeutic decisions at this time be individualized on a case by case basis [11]. Treatment should take into consideration the stage of disease, the gestational age at diagnosis, as well as preferences of the patient and her family. Fetal monitoring for fetal well-being and general development is recommended before each chemotherapy cycle when systemic therapy is initiated as the most common neonatal outcome associated with chemotherapy is intrauterine growth restriction. Adriamycin and cyclophosphamide are generally well tolerated in pregnancy and suggested to be two of the chemotherapy drugs of choice for treatment of PABC in a recent expert opinion published by Lambertini, et al. in which he reviews the literature to date which is admittedly limited of the safety profile of these drugs during pregnancy. The most common medical side effect of these drugs is myelosuppression in both the mother and fetus $[3,5,7,9,12,13]$. In one study, Rouzier demonstrated the chemosensitivity to these drugs was the same in tumors of $\mathrm{PABC}$ and tumors of non-pregnancy associated breast cancers $[3,14]$.

While anthracyclines, cyclophosphamides, and taxanes appear to be safe for use during pregnancy, anti-Her2 medications including Herceptin and endocrine therapies including tamoxifen are not. Several studies suggest that the use of Herceptin during pregnancy increases the risk to the fetus. It appears to be correlated with a higher incidence of oligohydramnios during pregnancy which can predispose to preterm labor and therefore increase fetal morbidity and mortality. Similarly, tamoxifen also increases the risk to the fetus specifically through anatomic defects including craniofacial malformations and ambiguous genitalia $[9,11,12]$. For these reasons, our patient was not given these classes of medications until after delivery.

Another interesting finding of this case involves the dose dense nature of the neoadjuvant chemotherapy regimen with G-CSF support. To our knowledge only one additional paper discussed this type of regimen during pregnancy. Published in 2012, Cardonick, et al. compared a small cohort of 10 women with PABC receiving dose dense chemotherapy every 2 weeks to a cohort of 99 women with PABC receiving conventional chemotherapy every 3 weeks. Their findings suggested that dose dense chemotherapy poses no additional risk to either maternal or neonatal outcomes with no statistically significant increases in neutropenia nor time to recurrence of cancer [15]. As in Cardonick's paper, our case also showed no evidence of neutropenia in mother or baby which is hypothesized to be the most significant factor that may discourage providers from offering a dose dense regimen during pregnancy.

Surgery has been suggested as the first-line treatment of pregnancyassociated breast cancer when found in the very early stages during early pregnancy $[11,13]$. However, given the increased risk of spontaneous abortion during the first trimester, many surgeons may prefer to wait until a later gestational age. Regardless of gestational age, most studies agree that breast reconstruction should be delayed until after delivery due to inherent changes that occur in the unaffected breast after pregnancy.

\section{Conclusion}

As stated by Loibl et al. in his review, the current suggestion is that breast cancer treatment during pregnancy should emulate treatment options offered to non-pregnant individuals. While there are currently no treatment guidelines put forth by the American College of Obstetrics and Gynecology (ACOG), our case illustrates a similar treatment pathway as suggested by Loibl:

Diagnosis by core biopsy and selection of surgical option is similar to those in non-pregnant women. Radiotherapy should be postponed until after delivery and sentinel node mapping with blue dye only and flap reconstruction should be avoided. Chemotherapy should be delayed until after the first trimester. Standard adjuvant/neoadjuvant anthracycline, cyclophosphamide, and taxane combinations should be used. Dose-dense but not intensified dose-dense therapy can be given with special attention paid and dose being determined according to actual weight. Individual risk/benefit analysis, taking into account bother mother and fetus, is crucial and that a multidisciplinary discussion was required. Delivery as close to term as possible is recommended, with a 2-3 weeks interval between the last chemotherapy cycle and delivery to allow the bone marrow to recover and prevent hematologic toxicity, which was what was done with our patient. The review and our case highlight how recent advances in breast cancer diagnosis and treatment can be adapted for pregnant patients [11].

The goal of treatment is always to maximize the potential for cure while at the same time minimizing cancer treatment risk to the fetus. Our patient strongly desired to carry the pregnancy to term (in an effort to decrease the risk of prematurity in the developing infant) and began treatment prior to delivery, making this case unique in comparison to other publications. More focus is needed on the pregnant women who desire to continue pregnancy while simultaneously starting treatment, and ACOG adapted guidelines are needed for appropriate fetal surveillance and delivery planning for these women. An informed decision with the best interest of both the mother and the developing fetus in mind has to be made in a multidisciplinary manner with active involvement of the patient throughout the entire process.

\section{References}

1. Amant F, Loibl S, Neven P, Van Calsteren K (2012) Breast cancer in pregnancy. Lancet 379: 570-579.

2. Amant F, von Minckwitz G, Han SN, Bontenbal M, Ring AE, et al. (2013) Prognosis of women with primary breast cancer diagnosed during pregnancy: results from an international collaborative study. J of Clin Oncol 31: 2532-2539.

3. Cardonick E (2014) Pregnancy-associated breast cancer: optimal treatment options. Int J Womens Health 6: 935-943. 
Citation: Grant J, DiCarlo C, Woessner-Hoyson J (2016) Importance of a Multidisciplinary Approach to Breast Cancer Treatment in Pregnancy: Case Report of New Diagnosis of Pregnancy-Associated Breast Cancer. J Carcinog Mutagen 7: 279. doi:10.4172/2157-2518.1000279

Page 4 of 4

4. Krishna I, Lindsay M (2013) Breast cancer in pregnancy. Obstet Gynecol Clin North Am 40: 559-571.

5. Kerr J (2005) Neonatal effects of breast cancer chemotherapy administered during pregnancy. Pharmacotherapy 25: 438-441.

6. Nargotra N, Kalita D (2015) Pregnancy Associated Breast Cancer: Awareness is the Key to Diagnosis - A Case Report. J Clin Diagn Res 9: ED09-11.

7. Sule EA, Ewemade F (2015) Management of pregnancy associated breast cancer with chemotherapy in a developing country. Int J Surg Case Rep 17: $117-120$

8. Shim MH, Mok CW, Chang KH, Sung JH, Choi SJ, et al. (2016) Clinical characteristics and outcome of cancer diagnosed during pregnancy. Obstet Gynecol Sci 59: 1-8.

9. Lambertini M, Kamal NS, Peccatori FA, Del Mastro L, Azim HA Jr (2015) Exploring the safety of chemotherapy for treating breast cancer during pregnancy. Expert Opin Drug Saf 14: 1395-1408.

10. Lambertini M, Peccatori FA, Azim HA Jr. (2015) Targeted agents for cancer treatment during pregnancy. Cancer Treat Rev 41: 301-309.
11. Loibl S, Schmidt A, Gentilini O, Kaufman B, Kuhl C, et al. (2015) Breast cancer diagnosed during pregnancy adapting recent advances in breast cancer care for pregnant patients. JAMA Oncol 1: 1145-1153.

12. Amant F, Vandenbroucke T, Verheecke M, Fumagalli M, Halaska MJ, et al. (2015) Pediatric Outcome after Maternal Cancer Diagnosed during Pregnancy. N Engl J Med 373: 1824-1834.

13. Zagouri F, Psaltopoulou T, Dimitrakakis C, Bartsch R, Dimopoulos MA (2013) Challenges in managing breast cancer during pregnancy. J Thorac Dis 5 Suppl 1: S62-67.

14. Rouzier R, Werkoff G, Uzan C, Mir O, Gligorov J, et al. (2011) Pregnancy-associated breast cancer is as chemosensitive as nonpregnancy-associated breast cancer in the neoadjuvant setting. Annals of Onc 22: 1582-1587.

15. Cardonick E, Gilmandyar D, Somer RA (2012) Maternal and neonatal outcomes of dose-dense chemotherapy for breast cancer in pregnancy. Obstet Gynecol 120: 1267-1272. 